\title{
ON COMPLEX SPACES WITH PRESCRIBED SINGULARITIES
}

\author{
M. Colţoiu, K. Diederich, C. Joiţa
}

To the memory of our unforgettable teacher and shining example Hans Grauert

\begin{abstract}
For a given complex space $Y$ we construct a complex space $X$ such that $\operatorname{Sing}(X)=Y$.
\end{abstract}

\section{Introduction}

For a reduced complex space $X$ we denote by $\operatorname{Sing}(X)$ the set of singular points of $X$. In this paper we are dealing with the following question: given a reduced complex space $Y$, does there exists a reduced complex space $X$ such that $\operatorname{Sing}(X)=Y$. We show that the answer is "yes". Namely we prove the following theorem:

Theorem 1. Let $Y$ be a reduced complex space. Then there exists a reduced complex space $X$ such that:

(1) $\operatorname{Sing}(X)=Y, \operatorname{dim}(X)=\operatorname{dim}(Y)+2$.

(2) along $\operatorname{Reg}(Y)$, the complex space $X$ has only quadratic singularities, (i.e., the product of a complex manifold of dimension $n=\operatorname{dim}(Y)$ and a surface with an isolated quadratic 2-dimensional singularity).

Moreover, if $Y$ is normal then $X$ can be chosen to be normal and if $Y$ is locally irreducible then $X$ can be chosen to be locally irreducible.

If $Y$ is a complex manifold the proof is trivial because one can choose $X=Y \times$ $S$ where $S$ has only one singular point. Obviously this argument does not work if $\operatorname{Sing}(Y) \neq \emptyset$ because $\operatorname{Sing}(Y \times S)=\operatorname{Sing}(Y) \times S \bigcup Y \times \operatorname{Sing}(S)$. To prove our main theorem we consider a resolution of singularities $\pi: \tilde{Y} \rightarrow Y$ (which exists by the results of Bierstone and Milmann [3], and Aroca, et al. [1]) and over $\tilde{Y}$ we consider a rank 2 vector bundle $E \rightarrow \tilde{Y}$, which is relatively negative. On each fiber of $E$ we have the equivalence relation $x \sim(-x)$. If we let $F:=E / \sim$ we obtain a locally trivial fibration $\tau: F \rightarrow \tilde{Y}$ with typical fiber $\left\{\left(z_{1}, z_{2}, z_{3}\right) \in \mathbb{C}^{3}: z_{1} z_{2}=z_{3}^{2}\right\}$, which has a quadratic two-dimensional isolated singularity. From $F$ we get the desired complex space $X$ by applying the relative Remmert quotient theorem (see [11]) and Wiegmann quotient theorem [15].

In the embedded case, i.e., if $Y$ is a complex subspace of a complex manifold $Z$, we give another construction of $X$ using only Wiegmann quotient theorem. In this particular case, we obtain:

Received by the editors July 3, 2012.

2000 Mathematics Subject Classification. 32C45, 32C15, 32C40.

Key words and phrases. singularities, desingularization, holomorphically convex space, Remmert reduction, relatively exceptional, blowing down. 
Theorem 2. Suppose that $Z$ is a complex manifold and $Y$ is a closed subspace of $Z$. Then there exists a complex space $X$ with the following properties:

(1) $\operatorname{Sing}(X)=Y$ and $\operatorname{dim}(X)=\operatorname{dim}(Z)+1$.

(2) $X$ is locally irreducible.

(3) The normalization of $X$ is smooth and therefore $X$ is not normal at any point of $Y$.

(4) If $Z$ is connected then $X$ is irreducible.

\section{Preliminaries}

Throughout this paper all complex spaces are assumed to be reduced.

We recall that a complex space $X$ is called holomorphically convex if the holomorphically convex hull of every compact subset is compact.

Definition 1. A holomorphic map of complex spaces $\pi: X \rightarrow S$ is called holomorphically convex if for any point $s \in S$ there exists an open neighborhood $U$ of $s$ such that $X(U):=\pi^{-1}(U)$ is holomorphically convex. If for any point $s$ we can find $U$ such that $X(U)$ is Stein then $\pi$ is called a Stein morphism.

Knorr and Schneider in [11] proved the following result:

Theorem 3. Suppose that $\pi: X \rightarrow S$ is a holomorphically convex map between two complex spaces. Then there exists a complex spaces $R$ and a holomorphic map $\rho: X \rightarrow R$, called the relative Remmert reduction of $\pi$, such that $\rho_{*} \mathcal{O}_{X}=\mathcal{O}_{R}$ (so $\rho$ is proper, surjective, and has connected fibers) and a commutative diagram

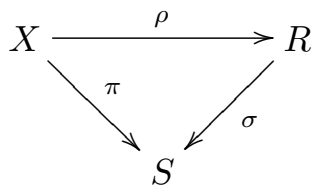

with $\sigma$ being a Stein morphism.

Throughout this paper a complex space $X$ is called 1-convex if there exists a smooth exhaustion function $\phi: X \rightarrow \mathbb{R}$ which is strictly plurisubharmonic outside a compact subset $K \subset X$.

Definition 2. A holomorphic map $\pi: X \rightarrow S$ is called 1-convex if for any $s \in S$ there exists an open neighborhood $U$ of $s$, a $\mathcal{C}^{\infty}$ function $\phi: X(U) \rightarrow \mathbb{R}$ and a real number $c_{0} \in \mathbb{R}$ such that:

(1) $\phi_{\mid\left\{x \in X(U): \phi(x)>c_{0}\right\}}$ is 1-convex,

(2) for every $c \in \mathbb{R}$ we have that $\pi_{\mid\{x \in X(U): \phi(x) \leq c\}}$ is a proper map.

The following Theorem is Satz. 3.4 in [11], see also [14].

Theorem 4. Every 1-convex map is holomorphically convex.

We recall the definition of a relatively exceptional set given in [11].

Definition 3. Suppose that $\pi: X \rightarrow S$ is a holomorphic map between two complex spaces and $A \subset X$ is a closed analytic subset such that $\pi_{\mid A}$ is proper and has nowhere 
discrete fibers. $A$ is called relatively exceptional with respect to $\pi$ if there exists a commutative diagram

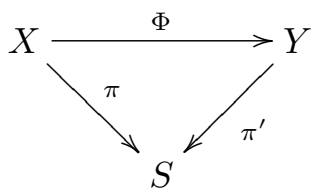

where $Y$ is a complex space and $\pi^{\prime}$ and $\Phi$ are holomorphic maps, such that:

(i) $\pi_{\mid \Phi(A)}^{\prime}$ has discrete fibers,

(ii) $\Phi$ induces a biholomorphism $X \backslash A \rightarrow Y \backslash \Phi(A)$,

(iii) $\Phi_{*}\left(\mathcal{O}_{X}\right)=\mathcal{O}_{Y}$.

Definition 4. If $\pi: X \rightarrow S$ is a holomorphic map between two complex spaces and $A$ is a closed analytic subset of $X$, then $A$ is called maximally proper over $S$ if $\pi_{\mid A}$ is proper, has nowhere discrete fibers and for any closed analytic subset $A^{\prime}$ of $X$ with these two properties we have $A^{\prime} \subset A$.

The following result is Satz 5.4 of [11].

Proposition 1. Suppose that $\pi: X \rightarrow S$ is a holomorphic map and $A \subset X$ is a closed analytic subspace of $X$. We assume that $A$ has a neighborhood $W$ such that $\pi_{\mid W}$ is 1-convex and $A$ is maximally proper over $S$ in $W$. Then $A$ is relatively exceptional with respect to $S$.

We identify a vector bundle with the sheaf of germs of local sections in the bundle. Suppose that $X$ is a compact complex space and $p: E \rightarrow X$ is a holomorphic vector bundle of rank $r$. We let $\pi: \mathbb{P}(E) \rightarrow X$ be the holomorphic fiber bundle for which $\pi^{-1}(x)$ is the space of all $(r-1)$-dimensional linear subspaces of $p^{-1}(x)$. In general, for a coherent sheaf $\mathcal{F}$ on $X$ one ca associate a projective variety over $X, \mathbb{P}(\mathcal{F})$, obtaining in this way a contravariant functor. For details we refer to [8] and [5], Chapter 1. For the proof of the following theorem see [7] and [10].

Theorem 5. The following statements are equivalent:

(a) $L=\mathcal{O}_{\mathbb{P}(E)}(1)$ is ample.

(b) For every coherent sheaf $\mathcal{F}$ on $X$ there exists a positive integer $m_{0}$ such that $H^{q}\left(X, \mathcal{F} \otimes S^{m}(E)\right)=0$ for every $q \geq 1, m \geq m_{0}\left(S^{m}(E)\right.$ denotes the $m$-th symmetric power of $E)$.

(c) For every coherent sheaf $\mathcal{F}$ on $X$ there exists a positive integer $m_{0}$ such that $\mathcal{F} \otimes S^{m}(E)$ is spanned by its global sections.

(d) The zero section of $E^{*}$ is exceptional.

(e) The zero section of $E^{*}$ has a strongly pseudoconvex neighborhood.

A vector bundle is called ample if the above equivalent conditions are satisfied. A vector bundle is called negative if its dual is ample.

We will need the following generalization in the relative case. Suppose that $\pi$ : $X \rightarrow S$ is a proper holomorphic map and $p: E \rightarrow X$ is a holomorphic vector bundle.

Definition 5. (a) $E$ is called relatively negative if its restriction to every fiber of $\pi^{-1}(s)$ is negative in the sense of Grauert, i.e., the null-section has a strictly pseudoconvex neighborhood. 
(b) $E$ is called relatively ample if its dual $E^{*}$ is relatively negative.

(c) $\pi: X \rightarrow S$ is called relatively ample if there exists a relatively ample line bundle $p: L \rightarrow X$.

For the next Lemma see Corollary 2.7 in [13]

Lemma 1. Suppose that $s_{0}$ is a point in $S$ and $E_{\mid \pi^{-1}\left(s_{0}\right)}$ is negative. Then there exists a neighborhood $U$ of $s_{0}$ such that $\pi \circ p$ is a 1-convex morphism on $p^{-1}\left(\pi^{-1}(U)\right)$.

Corollary 1. If $\pi$ has nowhere discrete fibers then $E$ is relatively negative iff its null-section is relatively exceptional.

Remark: For more general results concerning the relative blowing down of complex spaces, see [6].

Suppose now that $X$ and $Y$ are complex spaces, $f: X \rightarrow Y$ is a proper holomorphic map, and $L \rightarrow X$ a holomorphic line bundle. It was proved in [13], Theorem 3.6, (using the results on 1-convex morphisms obtained in [11]) that $L$ is relatively ample with respect to $f$ if and only if for every coherent sheaf $\mathcal{F}$ on $X$ and every compact set $K \subset Y$ there exists a positive integer $n_{0}=n_{0}(K, \mathcal{F})$ such that $R^{q} f_{*}(\mathcal{F}(n))=0$ on $K$ for every $n \geq n_{0}$ and every $q \geq 1\left(\mathcal{F}(n)\right.$ stands for $\left.\mathcal{F} \otimes L^{n}\right)$. At the same time in [2], chapter 4 , Théorème 4.1 , it was shown that this last property implies that for every point $y \in Y$ there exists a neighborhood $V$ of $y$ and a large enough positive integer $n$ such that, on $f^{-1}(V)$, the cannonical morphism $f^{-1}(V) \rightarrow \mathbb{P}\left(f_{*}\left(L^{n}\right)\right.$ is an embedding. Moreover, in the proof of this theorem of [2] (page 179) it was shown that by further increasing $n$ we obtain that for every relatively compact open subset $U$ of $Y$ the cannonical morphism $f^{-1}(U) \rightarrow \mathbb{P}\left(f_{*}\left(L^{n}\right)\right)$ is an embedding for $n$ large enough ( $n$ depending on $U$ ). Therefore putting together Theorem 3.6 in [13] and Theorem 4.1, chapter 4 in [2], when $X$ and $Y$ are compact, we have:

Theorem 6. If $X$ and $Y$ are compact complex spaces, $f: X \rightarrow Y$ is a holomorphic map, and $L \rightarrow X$ a holomorphic line bundle, the following are equivalent:

(a) $L$ is relatively ample with respect to $f$.

(b) There exists $n_{0}$ such that $R^{q} f_{*}(\mathcal{F}(n))=0$ for every $n \geq n_{0}$ and every $q \geq 1$.

(c) There exists $n_{0}$ such that the canonical morphism $f^{*} f_{*} \mathcal{F}(n) \rightarrow \mathcal{F}(n)$ is surjective for every $n \geq n_{0}$.

(d) There exists $n_{1}$ such that $X \rightarrow \mathbb{P}\left(f_{*}\left(L^{n}\right)\right)$ is an embedding for $n \geq n_{1}$.

Remark. From (c) we have an embedding $X \hookrightarrow \mathbb{P}\left(f^{*} f_{*} L^{n}\right)=\mathbb{P}\left(f_{*} L^{n}\right) \times_{Y} X$, hence a map $X \rightarrow \mathbb{P}\left(f_{*} L^{n}\right)$. Condition (d) means that increasing $n$ this map becomes an embedding.

The following Lemma is a folklore result (see e.g. [9] Exercise 5.12). For reader's convenience we provide a proof.

Lemma 2. Suppose that $X$ and $Y$ are compact complex spaces, $f: X \rightarrow Y$ a holomorphic map, $G \rightarrow Y$ an ample line bundle and $L \rightarrow X$ a relatively ample line bundle with respect to $f$. Then $L \otimes f^{*} G$ is ample on $X$. 
Proof. Using Theorem 6, we choose a positive integer $n$ such that we have an embedding $j$ over $Y$ :

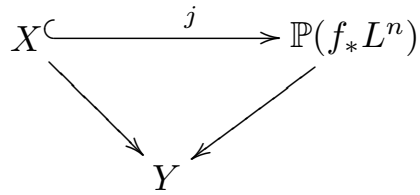

such that $L^{n}=j^{*}(\mathcal{O}(1))$. By [8], Proposition 1.5, if $\mathcal{F}_{1} \rightarrow \mathcal{F}_{2}$ is a sheaf epimorphism then one has an embedding $\mathbb{P}\left(\mathcal{F}_{2}\right) \hookrightarrow \mathbb{P}\left(\mathcal{F}_{1}\right)$ over $Y$, which is linear over each fiber. Since $G$ is ample it follows that, for $\nu$ large enough, $f_{*} L^{n} \otimes G^{\nu}$ is generated by global sections. Hence we have an epimorhism $\mathcal{O}_{Y}^{k} \longrightarrow f_{*} L^{n} \otimes G^{\nu}$ for some $k$. Because $G$ is a line bundle we have that $\mathbb{P}\left(f_{*} L^{n} \otimes G^{\nu}\right)=\mathbb{P}\left(f_{*} L^{n}\right)$. Passing to the associated projective spaces, we get an embedding $h: \mathbb{P}\left(f_{*} L^{n}\right) \hookrightarrow Y \times \mathbb{P}^{k-1}$ over $Y$ such that $\mathcal{O}(1)$ over $\mathbb{P}\left(f_{*} L^{n}\right)$ is the pull-back by $h$ of the hypersection bundle of $\mathbb{P}^{k-1}$. Composing with $j$ and using again the ampleness of $G$ we get that $L^{n} \otimes f^{*} G^{\mu}$ is ample for every $\mu$. In particular it is ample for $\mu=n$ and this in turn implies that $L \otimes f^{*} G$ is ample.

We will briefly recall some facts about desingularization of complex spaces (see [3]).

Let $X$ be a complex space and $Z \subset X$ a smooth closed complex subspace. For any point $x_{0} \in X$ we choose $U$ an open neighborhood of $x_{0}$ together with a closed embedding $U \hookrightarrow B \Subset \mathbb{C}^{N}$ where $B$ is an open ball in $\mathbb{C}^{N}$. Then $Z$ corresponds to a complex submanifold $W$ of $B$ and we consider the blow-up of $B$ with center $W$. In this blow-up we consider the proper transform of $U$ and in this way we obtain the blow-up of $U$ with center $U \cap Z$. This construction does not depend on the local embedding and the local blow-ups patch-up to get the blow-up of $X$ with (smooth) center $Z$.

The following result (Theorem 13.4 of [3]) is the fundamental theorem of global desingularization of complex spaces.

Theorem 7. Any complex space $X$ admits a desingularization $\pi: \tilde{X} \rightarrow X$ such that $\pi$ is the composition of a locally finite sequence of blow-ups with smooth centers and $\pi^{-1}(\operatorname{Sing}(X))$ is a divisor with normal crossings in $\tilde{X}$.

In this theorem locally finite means that on compact sets all but finitely many blow-ups are trivial.

Corollary 2. The desingularization $\pi: \tilde{X} \rightarrow X$ given by Theorem 7 is relatively ample, the relatively ample line bundle $p: L \rightarrow \tilde{X}$ corresponding to the exceptional divisor of $\pi$.

Proof. Let

$$
\cdots \rightarrow X_{3} \stackrel{\pi_{3}}{\longrightarrow} X_{2} \stackrel{\pi_{2}}{\longrightarrow} X_{1} \stackrel{\pi_{1}}{\longrightarrow} X
$$

be the sequence of blow-ups given by Theorem 7 and $L_{j} \rightarrow X_{j}$ the line bundle corresponding to the exceptional divisor of $\pi_{j}$. Each $L_{j}$ is relatively ample with respect to $\pi_{j}$.

Suppose that $x$ is a point in $X$. We consider the restrictions of $L_{1}$ and $L_{2}$ to $\pi_{1}^{-1}(x)$ and, respectively, $\left(\pi_{1} \circ \pi_{2}\right)^{-1}(x)$ and we denote them by $L_{1} \rightarrow \pi_{1}^{-1}(x)$ and $L_{2} \rightarrow\left(\pi_{1} \circ \pi_{2}\right)^{-1}(x)$. We have that $L_{1} \rightarrow \pi_{1}^{-1}(x)$ is ample and $L_{2} \rightarrow\left(\pi_{1} \circ \pi_{2}\right)^{-1}(x)$ 
is relatively ample with respect to $\pi_{2}$. We apply Lemma 2 and we deduce that $L_{2} \otimes \pi_{2}^{*}\left(L_{1}\right) \rightarrow\left(\pi_{1} \circ \pi_{2}\right)^{-1}(x)$ is ample.

We conclude that $L_{2} \otimes \pi_{2}^{*}\left(L_{1}\right) \rightarrow X$ is relatively ample with respect to $\pi_{1} \circ \pi_{2}$. We continue inductively this procedure and we obtain that the line bundle $L$ defined, by abuse of notation, by $L=\otimes_{i \in \mathbb{N}} L_{i} \rightarrow \tilde{X}$ is relatively ample with respect to $\pi$.

The infinite tensor product of line bundles (and the entire construction) makes sense since the sequence of blow-ups is locally finite.

Definition 6. ([15]) Suppose that $\left(X, \mathcal{O}_{X}\right)$ is a complex space, $F$ is a subset of $\mathcal{O}_{X}(X)$ and let $\phi_{F}: X \rightarrow \mathbb{C}^{F}, \phi_{F}(x)=(f(x))_{f \in F}$.

(a) $\left(X, \mathcal{O}_{X}\right)$ is called $F$-separable if $\phi_{F}$ is injective.

(b) $\left(X, \mathcal{O}_{X}\right)$ is called $F$-convex if $\phi_{F}$ is proper.

$F$-separable means that functions in $F$ separate the points of $X$ and $F$-convex means that for every discrete sequence $\left\{x_{n}\right\}$ in $X$ there exists a function $f \in F$ such that $\left\{\left|f\left(x_{n}\right)\right|\right\}$ is unbounded.

The following theorem, generalizing a result of Remmert, was proved by Wiegmann [15].

Theorem 8. Suppose that $\left(X, \mathcal{O}_{X}\right)$ is a reduced complex space and $F$ is a subalgebra of $\mathcal{O}_{X}(X)$ such that $\left(X, \mathcal{O}_{X}\right)$ is $F$-convex. Then there exists an $F$-convex and $F$-separable reduced Stein space $\left(Y, \mathcal{O}_{Y}\right)$ together with a proper surjective holomorphic mapping $p:\left(X, \mathcal{O}_{X}\right) \rightarrow\left(Y, \mathcal{O}_{Y}\right)$ such that if $\pi: \mathcal{O}_{Y}(Y) \rightarrow \mathcal{O}_{X}(X)$ is the induced morphisms of $\mathbb{C}$-algebras then $\pi\left(\mathcal{O}_{Y}(Y)\right) \supset F$. Moreover, $\left(Y, \mathcal{O}_{Y}\right)$ is unique, up to isomorphism, with these properties, if $F$ is closed in $\mathcal{O}_{X}(X)$ then $\pi\left(\mathcal{O}_{Y}(Y)\right)=F$ and if $F=\mathcal{O}_{X}(X)$ then $\pi$ is an isomorphism.

The complex space $\left(Y, \mathcal{O}_{Y}\right)$ is called the Remmert reduction of $\left(X, \mathcal{O}_{X}\right)$ with respect to $F$ and is denoted by $R_{F}\left(X, \mathcal{O}_{X}\right)$. Note that Remmert's theorem corresponds to the case $F=\mathcal{O}_{X}(X)$.

For a complex space $\left(Z, \mathcal{O}_{Z}\right)$ we let $T\left(Z, \mathcal{O}_{Z}\right)$ be the underlying topological space $Z$ and, for an open subset $U$ of $Z, \Gamma_{U}\left(Z, \mathcal{O}_{Z}\right)=\mathcal{O}_{Z}(U)$. We recall briefly Wiegmann's construction. The topological space $T\left(R_{F}\left(X, \mathcal{O}_{X}\right)\right)$ is defined as $T\left(R_{F}\left(X, \mathcal{O}_{X}\right)\right)=$ $X / \sim$ and $p$ is the quotient map, where, for $x_{1}, x_{2} \in X, x_{1} \sim x_{2}$ if and only if $f\left(x_{1}\right)=f\left(x_{2}\right)$ for every $f \in F$. The structure sheaf is defined as follows. For $y \in$ $T\left(R_{F}\left(X, \mathcal{O}_{X}\right)\right)$ let $m_{y}$ by the ideal of $F$ that contains all function $f \in F$ that vanish on $p^{-1}(y)$. For every open subset $U$ of $T\left(R_{F}\left(X, \mathcal{O}_{X}\right)\right), \Gamma_{U}\left(R_{F}\left(X, \mathcal{O}_{X}\right)\right)$ is the algebra of all functions $g \in \mathcal{O}_{X}\left(p^{-1}(U)\right)$ such that for every point $y \in U$ there exists a positive integer $k$, a convergent power series $\sum_{i_{1}, \ldots, i_{k}}^{\infty} c_{i_{1}, \ldots, i_{k}} T_{1}^{i_{1}} \ldots T_{k}^{i_{k}} \in \mathbb{C}\left[\left\langle T_{1}, \ldots, T_{k}\right\rangle\right]$ and $f_{1}, \ldots, f_{k} \in m_{y}$ such that $\sum_{i_{1}, \ldots, i_{k}}^{\infty} c_{i_{1}, \ldots, i_{k}} f_{1}^{i_{1}} \cdots f_{k}^{i_{k}}$ converges uniformly to $g$ on a neighborhood of $p^{-1}(y)$.

Lemma 3. Suppose that $\left(X, \mathcal{O}_{X}\right)$ is a reduced complex space, $F$ and $G$ are two subalgebras of $\mathcal{O}_{X}(X)$ such that $\left(X, \mathcal{O}_{X}\right)$ is $F$-convex, $F \subset G$ and $F$ is dense in $G$. Then the canonical morphism $R_{F}\left(X, \mathcal{O}_{X}\right) \rightarrow R_{G}\left(X, \mathcal{O}_{X}\right)$ is an isomorphism.

Proof. It follows from the discussion after Theorem 8 that if $F$ is a dense subset of $G$ then $T\left(R_{F}\left(X, \mathcal{O}_{X}\right)\right)=T\left(R_{G}\left(X, \mathcal{O}_{X}\right)\right)$ and that for every open subset $U$ of $T\left(R_{F}\left(X, \mathcal{O}_{X}\right)\right)$ we have $\Gamma_{U}\left(R_{F}\left(X, \mathcal{O}_{X}\right)\right) \subset \Gamma_{U}\left(R_{G}\left(X, \mathcal{O}_{X}\right)\right)$. 
Let $\bar{F}$ be the closure of $F$ (hence $G \subset \bar{F}$ ) and let $Y:=T\left(R_{F}\left(X, \mathcal{O}_{X}\right)\right)$. We have then $F \subset \Gamma_{Y}\left(R_{F}\left(X, \mathcal{O}_{X}\right)\right) \subset \Gamma_{Y}\left(R_{G}\left(X, \mathcal{O}_{X}\right)\right) \subset \Gamma_{Y}\left(R_{\bar{F}}\left(X, \mathcal{O}_{X}\right)\right)=\bar{F}$. As $\Gamma_{Y}\left(R_{F}\left(X, \mathcal{O}_{X}\right)\right)$ and $\Gamma_{Y}\left(R_{G}\left(X, \mathcal{O}_{X}\right)\right)$ are closed in $\mathcal{C}(Y)$ (the algebra of continuous functions on $Y$ ) and $\bar{F}$ is the smallest closed subset containing $F$, it follows that the map $\Gamma_{Y}\left(R_{F}\left(X, \mathcal{O}_{X}\right)\right) \rightarrow \Gamma_{Y}\left(R_{G}\left(X, \mathcal{O}_{X}\right)\right)$ is bijective. As both $R_{F}\left(X, \mathcal{O}_{X}\right)$ and $R_{G}\left(X, \mathcal{O}_{X}\right)$ are reduced Stein spaces it follows that the canonical morphism $R_{F}\left(X, \mathcal{O}_{X}\right) \rightarrow R_{G}\left(X, \mathcal{O}_{X}\right)$ is an isomorphism.

In Wiegmann's theorem one needs $X$ to be $F$-convex. In particular, $X$ has to be $\mathcal{O}_{X}(X)$-convex which is a strong global condition. On the other hand, it may happen that $\mathcal{O}_{X}(X)=\mathbb{C}$ (e.g., if $X$ is compact) and then the Remmert reduction is just a point. For our purpose we need to apply Wiegmann's theorem locally. To be able do this, we need a "patching" result. This is the purpose of the following proposition.

Proposition 2. Suppose that $\left(X, \mathcal{O}_{X}\right)$ is a reduced complex space and $\left\{V_{i}\right\}_{i \in \mathbb{N}}$ is a locally finite open covering of $X$. Let $F_{i}$ be a closed subalgebra of $\mathcal{O}_{X}\left(V_{i}\right), \sim_{i}$ be the equivalence relation on $V_{i}$ induced by $F_{i}\left(x_{1} \sim_{i} x_{2}\right.$ iff $\left.f\left(x_{1}\right)=f\left(x_{2}\right) \forall f \in F_{i}\right)$, and $F_{i j}=F_{j i}$ be a closed subalgebra of $\mathcal{O}_{X}\left(V_{i} \cap V_{j}\right)$. We assume that:

(a) $\mathcal{O}_{X} \mid V_{i}$ is $F_{i}$-convex,

(b) $F_{i \mid V_{i} \cap V_{j}}$ is a dense subset of $F_{i j}$ for every $i, j \in \mathbb{N}$,

(c) $V_{i} \cap V_{j}$ is saturated with respect to $\sim_{i}$ for every $i, j \in \mathbb{N}$.

Then there exists a reduced complex space $\left(Y, \mathcal{O}_{Y}\right)$, a proper holomorphic map $p$ : $X \rightarrow Y$ and an open covering $\left\{U_{i}\right\}_{i}$ of $Y$ such that $\left(U_{i}, \mathcal{O}_{Y \mid U_{i}}\right)$ is isomorphic to $R_{F_{i}}\left(V_{i}, \mathcal{O}_{X} \mid V_{i}\right)$ and $p_{\mid U_{i}}$ is the canonical morphism given by Theorem 8.

Proof. We define the following relation on $X: x \sim y$ if and only if there exists $i \in \mathbb{N}$ such that $x, y \in V_{i}$ and $x \sim_{i} y$. Note that if $x \in V_{i}, y \in V_{i} \cap V_{j}$ and $x \sim_{i} y$ then using (c) we get that $x \in V_{i} \cap V_{j}$ and by (b) and Lemma 3 we get that $x \sim_{j} y$. This shows that $\sim$ is an equivalence relation. Moreover, each $V_{i}$ is saturated with respect to $\sim$. Let $Y=X / \sim$, endowed with the quotient topology, and $p: X \rightarrow Y$ be the quotient map. We set $U_{i}=p\left(V_{i}\right)$ which is an open subset of $Y$. By Wiegmann's construction of $R_{F_{i}}\left(V_{i}, \mathcal{O}_{X} \mid V_{i}\right)$ explained above we have that $T\left(R_{F_{i}}\left(V_{i}, \mathcal{O}_{X} \mid V_{i}\right)\right)=U_{i}$. We define the structure sheaf $\mathcal{O}_{Y}$ as follows: if $\Omega$ is an open subset of $Y$ and $f \in \mathcal{C}(\Omega)$ then $f \in \mathcal{O}_{Y}(\Omega)$ if and only if for every point $y \in U_{i}$ for some $i \in I$ there exists $D$ an open subset of $Y$ such that $D \subset \Omega \cap U_{i}$ and $f_{\mid D} \in \Gamma_{D}\left(R_{F_{i}}\left(V_{i}, \mathcal{O}_{X} \mid V_{i}\right)\right)$. By Lemma 3 this definition does not depend on the choice of $i$. The fact that $\left(U_{i}, \mathcal{O}_{Y \mid U_{i}}\right)$ is isomorphic to $R_{F_{i}}\left(V_{i}, \mathcal{O}_{X} \mid V_{i}\right)$ follows from the construction of the relative Remmert reduction.

Example. Suppose that $X=\mathbb{P}^{1}$. Let $B_{1}, B_{2}, B_{3}$ be three balls (in local coordinate charts) such that $B_{1} \cup B_{2} \cup B_{3}=\mathbb{P}^{1}$ and $B_{i} \cap B_{j}$ is Runge in $B_{i}$ for every $i, j \in\{1,2,3\}$. We assume that $a:=[0: 1] \in B_{1} \backslash\left(\bar{B}_{2} \cup \bar{B}_{3}\right)$. Let $F_{2}=F_{22}=\mathcal{O}\left(B_{2}\right), F_{3}=F_{33}=$ $\mathcal{O}\left(B_{3}\right), F_{1}=F_{11}=\left\{f \in \mathcal{O}\left(B_{1}\right): f^{\prime}(a)=0\right\}$ and, for $i \neq j, F_{i, j}=\mathcal{O}\left(B_{i} \cap B_{j}\right)$. Then we are in the hypothesis of Proposition 2. Note that a holomorphic function $f$, defined in a neighborhood of the origin $0 \in \mathbb{C}$, satisfies $f^{\prime}(0)=0$ if and only if there exists a holomorphic function $F$ of two variables, defined in a neighborhood of the origin in $\mathbb{C}^{2}$, such that $f(z)=F\left(z^{3}, z^{2}\right)$ and the map $z \rightarrow\left(z^{3}, z^{2}\right)$ is a parameterization of the cusp singularity $\left\{(x, y) \in \mathbb{C}^{2}: x^{2}=y^{3}\right\}$. 
We deduce that the complex space that we obtain by applying Proposition 2 is $Y=\left\{\left[z_{0}: z_{1}: z_{2}\right] \in \mathbb{P}^{2}: z_{0}^{2} z_{2}=z_{1}^{3}\right\}$ and $p: \mathbb{P}^{1} \rightarrow Y$ is given by $p\left(\left[x_{0}: x_{1}\right]\right)=\left[x_{0}^{3}:\right.$ $\left.x_{0}^{2} x_{1}: x_{1}^{3}\right]$.

\section{The results}

Lemma 4. If $X$ is a complex space then any open covering has a locally finite open refinement $\left\{\Omega_{m}\right\}_{m \in \mathbb{N}}$ such that $\Omega_{m}$ is Stein for every $m \in \mathbb{N}$ and the pair $\left(\Omega_{m_{1}}, \Omega_{m_{1}} \cap\right.$ $\Omega_{m_{2}}$ ) is Runge for every $m_{1}, m_{2} \in \mathbb{N}$.

Proof. We consider $\left\{W_{j}\right\}_{j \in \mathbb{N}},\left\{V_{j}\right\}_{j \in \mathbb{N}},\left\{U_{j}\right\}_{j \in \mathbb{N}}$ locally finite countable open covering of $X$ such that $\left\{U_{j}\right\}_{j \in \mathbb{N}}$ is a refinement of the given covering, $W_{j} \Subset V_{j} \Subset U_{j}$ and $U_{j}$ is Stein for every $j \in \mathbb{N}$. For each $j \in \mathbb{N}$ and each $x \in \bar{W}_{j}$ we choose $\phi_{j, x}: U_{j} \rightarrow[0, \infty)$ a plurisubharmonic function such that:

(a) $\phi_{j, x}(x)=0$ and $\left\{z \in U_{j}: \phi_{j, x}(z)<1\right\} \subset V_{j}$,

(b) if, for some $k \in \mathbb{N},\left\{z \in U_{j}: \phi_{j, x}(z)<1\right\} \cap \bar{V}_{k} \neq \emptyset$ then $\left\{z \in U_{j}: \phi_{j, x}(z)<\right.$ $1\} \subset U_{k}$.

Then $\left\{z \in U_{j}: \phi_{j, x}(z)<1\right\}_{x \in \bar{W}_{j}}$ is an open covering of $\bar{W}_{j}$. We extract a finite subcovering $\left\{z \in U_{j}: \phi_{j, s}(z)<1\right\}_{s \in A_{j}}$ where $A_{j}$ is a finite set and we set $\Omega_{j, s}:=$ $\left\{z \in U_{j}: \phi_{j, s}(z)<1\right\}$. The $\left\{\Omega_{j, s}\right\}_{j, s}$ is a locally finite open covering of $X$. Since $\phi_{j, x}$ is plurisubharmonic on $U_{j}$ each $\Omega_{j, s}$ is Stein. On the other hand, if $\Omega_{j, s} \cap \Omega_{k, l} \neq \emptyset$, as $\Omega_{k, l} \subset V_{k}$, we have that $\Omega_{j, s} \cap V_{k} \neq \emptyset$ and hence by property (b) above we have that $\Omega_{j, s} \subset U_{k}$ This implies that $\Omega_{j, s} \cap \Omega_{k, l}=\left\{z \in \Omega_{j, s}: \phi_{k, l}(z)<1\right\}$ which is Runge in $\Omega_{j, s}$, see [12]. If we choose a bijection $\chi: \mathbb{N} \rightarrow\left\{(j, s): j \in \mathbb{N}, s \in A_{j}\right\}$ and we set $\Omega_{m}:=\Omega_{\chi(m)}$ we get the desired family.

Proof of Theorem 1. Let $\nu: Y_{1} \rightarrow Y$ be the normalization map and $\tau: Z \rightarrow Y_{1}$ be a desingularization map which is relatively ample. Let $p: L \rightarrow Z$ be a relatively negative line bundle (which exists by Corollary 2)and set $E:=L \oplus L$.

Let $\sigma: \mathbb{C}^{2} \rightarrow \mathbb{C}^{2}, \sigma(w)=-w$. Clearly $\sigma \circ \sigma$ is the identity of $\mathbb{C}^{2}$ and therefore we obtain a linear action of $\mathbb{Z}_{2}$ on $\mathbb{C}^{2}$. It is easy to see that $\mathbb{C}^{2} / \mathbb{Z}_{2}$ is isomorphic to $\left\{\left(z_{1}, z_{2}, z_{3}\right) \in \mathbb{C}^{3}: z_{1} z_{2}=z_{3}^{2}\right\}$ which is a normal surface with only one singular point of quadratic type. By linearity we obtain an action of $\mathbb{Z}_{2}$ on any vector bundle and in particular on the vector bundle $E$ defined above. Let $\tilde{E}$ be the quotient space of $E$ through this action. We get then a locally trivial fibration $\tilde{p}: \tilde{E} \rightarrow Z$ with typical fiber $\left\{\left(z_{1}, z_{2}, z_{3}\right) \in \mathbb{C}^{3}: z_{1} z_{2}=z_{3}^{2}\right\}$. Note that $\operatorname{Sing}(\tilde{E})=Z$ (the zero section). The composition $f:=\tau \circ \tilde{p}: \tilde{E} \rightarrow Y_{1}$ is 1-convex, and hence is a holomorphically convex map. Thus we can consider the relative Remmert quotient associated to $f$. We obtain a complex space $W_{1}$ together with a map $g: \tilde{E} \rightarrow W_{1}$ such that $g_{*} \mathcal{O}_{\tilde{E}}=\mathcal{O}_{W_{1}}$. We get then a closed embedding $\sigma: Y_{1} \hookrightarrow W_{1}$. Via this embedding $Y_{1}$ is the image through $g$ of the null-section of $\tilde{E}$. Note that $g$ is biholomorphic outside the nullsection and hence $W_{1}$ has singularities precisely on $Y_{1}$. There is a natural holomorphic retraction $r: W_{1} \rightarrow Y_{1}$, which is a Stein morphism, corresponding to the projection map $f: \tilde{E} \rightarrow Y_{1}$. Over the regular part of $Y_{1}$ the space $W_{1}$ has only quadratic singularities.

At this moment we reduced the proof of Theorem 1 to the following Lemma (relative contraction for finite maps), which will be applied to the normalization map. 
Lemma 5. Let $A$ and $B$ be complex spaces and $m: A \rightarrow B$ be a finite surjective holomorphic map. We assume that $A$ is a closed complex space of a complex space $S$ and $m$ admits a holomorphic extension $\tilde{m}: S \rightarrow B$ which is a Stein morphism. Then there exists a complex space $T$ and a holomorphic map $\alpha: S \rightarrow T$ such that $T$ contains $B$ as a closed complex subspace, $\alpha_{\mid A}=m$ and, outside $B, \alpha$ is a biholomorphism between $S \backslash A$ and $T \backslash B$.

Proof. Using Lemma 4 we choose a locally finite Stein covering $\left\{D_{i}\right\}_{i \in \mathbb{N}}$ of $B$ such that $D_{i} \cap D_{j}$ is Runge in $D_{i}$ and in $D_{j}$ for every $i, j \in \mathbb{N}$ and $\tilde{m}^{-1}\left(D_{i}\right) \subset S$ is Stein. Therefore $\tilde{m}^{-1}\left(D_{i} \cap D_{j}\right)$ is Runge in $\tilde{m}^{-1}\left(D_{i}\right)$ and in $\tilde{m}^{-1}\left(D_{j}\right)$ for every $i, j \in \mathbb{N}$. On $\tilde{m}^{-1}\left(D_{i}\right)$ we consider the set $F_{i}$ of all holomorphic functions $f \in \mathcal{O}\left(\tilde{m}^{-1}\left(D_{i}\right)\right)$ such that $f_{\mid A \cap \tilde{m}^{-1}\left(D_{i}\right)}$ comes from a holomorphic function on $D_{i}$, i.e., there exists a holomorphic function $g \in \mathcal{O}\left(D_{i}\right)$ with $f_{\mid A \cap \tilde{m}^{-1}\left(D_{i}\right)}=g \circ m$. Then $F_{i}$ is a subalgebra of $\mathcal{O}\left(\tilde{m}^{-1}\left(D_{i}\right)\right)$ and $\tilde{m}^{-1}\left(D_{i}\right)$ is $F_{i}$-holomorphically convex. Similarly, we define the set $F_{i j}$ of all holomorphic functions $f \in \mathcal{O}\left(\tilde{m}^{-1}\left(D_{i} \cap D_{j}\right)\right)$ such that $f_{\mid A \cap \tilde{m}^{-1}\left(D_{i} \cap D_{j}\right)}$ comes from a holomorphic function on $D_{i} \cap D_{j}$. Applying Wiegmann quotient theorem to the subalgebras $F_{i}$ we get a Stein complex space $T_{i}$ containing $D_{i}$ as a closed complex subspace. Using Proposition 2, these complex spaces $\left\{T_{i}\right\}_{i \in \mathbb{N}}$ can be glued together and we get the desired complex space $T$. This concludes the proof of Lemma 5 and of Theorem 1.

Proof of Theorem 2. Suppose that $\Omega$ is a Stein manifold and $A$ is a closed analytic subset of $\Omega$. We denote by $\pi: \Omega \times \mathbb{C} \rightarrow \Omega$ the standard projection and we identify a holomorphic function $f \in \mathcal{O}(\Omega)$ with $f \circ \pi$. Hence we have $\mathcal{O}(\Omega) \subset \mathcal{O}(\Omega \times \mathbb{C})$. Let $\lambda$ be the coordinate function on $\mathbb{C}$ and $F:=\left\{f \in \mathcal{O}(\Omega \times \mathbb{C}): \frac{\partial f}{\partial \lambda} \equiv 0\right.$ on $\left.A \times\{0\}\right\}$. Then:

- $F$ is a closed subalgebra of $\mathcal{O}(\Omega \times \mathbb{C})$ and $F \supset \mathcal{O}(\Omega)$,

- if $f \in \mathcal{O}(\Omega \times \mathbb{C})$ and $f_{\mid A \times\{0\}} \equiv 0$ then $f^{2} \in F$.

Suppose that $K$ is a compact subset of $\Omega \times \mathbb{C}$. Then $\widehat{K}^{F}$, the holomorphically convex

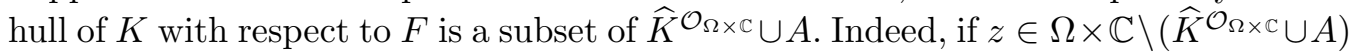
then there exists $f \in \mathcal{O}_{\Omega \times \mathbb{C}}$ such that $f_{\mid A \times\{0\}} \equiv 0$ and $|f(z)|>\|f\|_{K}$. It follows that $\left|f^{2}(z)\right|>\left\|f^{2}\right\|_{K}$ and $f^{2} \in F$. At the same time from $\mathcal{O}(\Omega) \subset F$ we get that

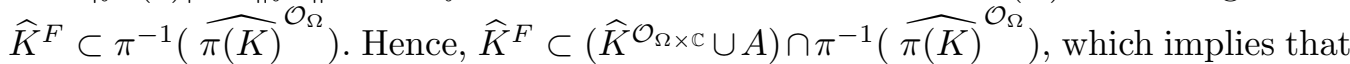
$\widehat{K}^{F}$ is compact and hence $\Omega \times \mathbb{C}$ is $F$-convex.

Similarly, we can show that $\Omega \times \mathbb{C}$ is $F$-separable. Namely, for any two points $x, y \in \Omega \times \mathbb{C}$, if $x, y \in A \times\{0\}$ then we can choose $f \in \mathcal{O}(\Omega)$ with $f(x) \neq f(y)$ and if at least one of them is not in $A$ we can choose $f \in \mathcal{O}(\Omega \times \mathbb{C}))$ such that $f^{2}(x) \neq f^{2}(y)$. Let $\left(Y, \mathcal{O}_{Y}\right)=R_{F}\left(\Omega \times \mathbb{C}, \mathcal{O}_{\Omega \times \mathbb{C}}\right), p: \Omega \times \mathbb{C} \rightarrow Y$ the canonical morphism and $B=p(A \times\{0\})$, which is a closed analytic subset of $Y$. Since $\Omega \times \mathbb{C}$ is $F$-separable it follows that $p$ is a homeomorphism.

We want to show next that $p: \Omega \times \mathbb{C} \backslash A \times\{0\} \rightarrow Y \backslash B$ is a biholomorphism and hence, in particular $\operatorname{Sing}(Y) \subset B$. It suffices to show that for any open subset $U$ of $\Omega \times \mathbb{C} \backslash A \times\{0\}$ and any $x \in U$ we have that every holomorphic function $f$ on $U$ can be approximated, uniformly on a neighborhood of $x$ by functions in $F$ (this will imply that the functions in $F$ give local coordinates outside $A \times\{0\})$. Let $c \in \mathbb{C}$ be such that $f(x)+c \neq 0$. We choose an open neighborhood $V$ of $x$ such that $V \Subset U$, 
$\bar{V} \cap A=\emptyset, \bar{V}$ is holomorphically convex and there exists a holomorphic function $g$ defined on a neighborhood of $\bar{V}$ such that $g^{2}=f+c$. It follows that we can find $\left\{h_{j}\right\}_{j \geq 0}, h_{j} \in \mathcal{O}(\Omega)$ such that $h_{j \mid A \times\{0\}} \equiv 0$ and $h_{j} \rightarrow g$ uniformly on $\bar{V}$. It remains to notice that $h_{j}^{2}-c \in F$ and $h_{j}^{2}-c \rightarrow f$ uniformly on $\bar{V}$.

Note also that $F \supset \mathcal{O}(\Omega)$ implies that $p_{\mid \Omega \times\{0\}}: \Omega \times\{0\} \rightarrow p(\Omega \times\{0\})$ is a biholomorphism and hence $p_{\mid A}: A \rightarrow B$ is a biholomorphism.

We claim now that $B \subset \operatorname{Sing}(Y)$. Let $y \in B$ and $x=p^{-1}(y) \in A$. If $Y$ were smooth in $y$, it would be normal in $y$, hence it would be normal in a neighborhood of $y$, and therefore we could find $U \subset X$ an open neighborhood of $x$ and $W \subset Y$ an open neighborhood of $y$ such that $p(U)=W$ and $p: U \rightarrow W$ is a biholomorphism. Therefore for every holomorphic function $f: U \rightarrow \mathbb{C}$ we would have that $f \circ p^{-1}$ is holomorphic on $W$. This would imply that we can approximate $f$, uniformly on a neighborhood of $x$, with functions from $F$. However, the coordinate function $\lambda: U \rightarrow$ $\mathbb{C}$ does not satisfy this property.

Lemma 6. Let $M$ be a Stein manifold, $A \subset M$ a closed analytic subset and $U \subset M a$ Runge open subset of $M$. Then the restriction map $f \rightarrow f_{\mid U \times \mathbb{C}}$ from $\{f \in \mathcal{O}(M \times \mathbb{C})$ : $\frac{\partial f}{\partial \lambda} \equiv 0$ on $\left.A \times\{0\}\right\}$ to $\left\{f \in \mathcal{O}(U \times \mathbb{C}): \frac{\partial f}{\partial \lambda} \equiv 0\right.$ on $\left.A \cap U \times\{0\}\right\}$ has dense image in the topology of uniform convergence on compacts. Here, $\lambda$ is the coordinate function on $\mathbb{C}$.

Proof. Let $f: U \times \mathbb{C} \rightarrow \mathbb{C}$ be a holomorphic function such that $\frac{\partial f}{\partial \lambda} \equiv 0$ on $A \cap U \times\{0\}$. Because $U \times \mathbb{C}$ is Runge in $M \times \mathbb{C}$ there exists a sequence of holomorphic functions $\left\{g_{n}\right\}_{n \geq 1}, g_{n} \in \mathcal{O}(M \times \mathbb{C})$, such that $g_{n} \equiv 0$ on $A \cap U \times\{0\}$ and $\left\{g_{n \mid U \times \mathbb{C}\}_{n \geq 1} \text { converges }}\right.$ to $\frac{\partial f}{\partial \lambda}$. At the same time there exists a sequence $\left\{h_{n}\right\}_{n \geq 1}, h_{n} \in \mathcal{O}(M)$ such that $\left\{h_{n \mid U}\right\}_{n \geq 1}$ converges to $f(z, 0)$. For each $n \geq 1$ we consider the following primitive with respect to $\lambda$ of $g_{n}: f_{n}(z, \lambda)=\int_{\gamma} g_{n}(z, \xi) \mathrm{d} \xi+h_{n}(z)$, where $\gamma:[0,1] \rightarrow \mathbb{C}$ is a path that joins $0 \in \mathbb{C}$ with $\lambda$. For $\gamma(t)=t z$ we get $f_{n}(z, \lambda)=\int_{0}^{1} g_{n}(z, t \lambda) \lambda \mathrm{d} t+h_{n}(z)$. We have then $\frac{\partial f_{n}}{\partial \lambda}=g_{n} \equiv 0$ on $A \times\{0\}$. At the same time, since both $f$ and $\int_{0}^{1} \frac{\partial f}{\partial \lambda}(z, t \lambda) \lambda \mathrm{d} t$ are primitives for $\frac{\partial f}{\partial \lambda}$, we have $f(z, \lambda)=\int_{0}^{1} \frac{\partial f}{\partial \lambda}(z, t \lambda) \lambda \mathrm{d} t+f(z, 0)$. Hence

$$
f_{n}(z, \lambda)-f(z, \lambda)=\int_{0}^{1}\left(g_{n}(z, t \lambda)-\frac{\partial f}{\partial \lambda}(z, t \lambda)\right) \lambda \mathrm{d} t+\left(h_{n}(z)-f(z, 0)\right) .
$$

Now, if $K \subset M \times C$ is a compact set, we choose $K_{0}$, a compact subset of $M$, and $B \subset \mathbb{C}$ a compact disk centered at the origin such that $K \subset K_{0} \times B$. Using $\left\|g_{n}-\frac{\partial f}{\partial \lambda}\right\|_{K_{0} \times B} \rightarrow 0$ and $\left\|h_{n}-f(z, 0)\right\|_{K_{0}} \rightarrow 0$, we obtain easily that $\left\|f_{n}-f\right\|_{K} \rightarrow 0$.

Let now $Z$ be a complex manifold and $Y$ a closed complex subspace of $Z$. We use Lemma 4 and we choose an open Stein covering $\left\{\Omega_{i}\right\}_{i \in \mathbb{N}}$ of $Z$ such that the pair $\left(\Omega_{i}, \Omega_{i} \cap \Omega_{j}\right)$ is Runge for every $i, j \in \mathbb{N}$. Let $F_{i}:=\left\{f \in \mathcal{O}\left(\Omega_{i} \times \mathbb{C}\right): \frac{\partial f}{\partial \lambda} \equiv 0\right.$ on $\left.Y \times\{0\}\right\}$ and, similarly, $F_{i j}:=\left\{f \in \mathcal{O}\left(\left(\Omega_{i} \cap \Omega_{j}\right) \times \mathbb{C}\right): \frac{\partial f}{\partial \lambda} \equiv 0\right.$ on $\left.Y \times\{0\}\right\}$.

We apply Wiegmann's quotient theorem to $F_{i}$ and we use Proposition 2, to glue together the complex spaces thus obtained and we get the desired complex space $X$. Note that because a positive codimension analytic subset does not disconnect a complex manifold it follows that $X$ is locally irreducible and, if $Z$ is connected, $X$ is 
irreducible. At the same time it follows from our proof that the normalization of $X$ is $Z \times \mathbb{C}$.

\section{Remarks:}

(1) In [4] the following result was proved : given a closed analytic subset $A$ of $\mathbb{C}^{n}, \operatorname{codim}(A) \geq 2$, there exists an irreducible analytic hypersurface $H \subset \mathbb{C}^{n}$ such that $\operatorname{Sing}(H)=A$. This shows, in particular, that one can prescribe singularities for Stein spaces. However the construction in [4] cannot be used for arbitrary singularities since it is not functorial and the local models cannot be glued together to obtain a complex space with prescribed singularities.

(2) The following problem was raised to the first author by C. Bănică in connection with the duality on complex spaces: could every complex space $Z$ of bounded Zariski dimension be embedded as a closed analytic subset of a complex manifold?

(3) The following problem remains open: suppose that $Y$ is a reduced complex space, not necessarily normal. Is it possible to find a normal complex space $X$ such that $\operatorname{Sing}(X)=Y$ ?

(4) If $Y$ is a projective algebraic variety then one can construct a normal projective algebraic variety $X$ such that $\operatorname{Sing}(X)=Y$. We would like to thank Iustin Coandă for this remark.

\section{Acknowledgments}

We would like to thank the referee for very useful remarks. The first and third named authors were supported by CNCS grant PN-II-ID-PCE-2011-3-0269.

\section{References}

[1] J.M. Aroca, H. Hironaka and J.L. Vicente, Desingularization theorems, (Mem. Math. Inst. Jorge Juan, No. 30) Madrid: Consejo Superior de Investigaciones Científicas 1977.

[2] C. Bănică and O. Stănăşilă, Méthodes algébriques dans la théorie globale des espaces complexes. Troisième édition. Gauthier-Villars, Paris, 1977.

[3] E. Bierstone and P.D. Milman, Canonical desingularization in characteristic zero by blowing up the maximum strata of a local invariant, Invent. Math. 128(2) (1997), 207-302.

[4] J.-P. Demailly, Construction d'hypersurfaces irréductibles avec lieu singulier donné dans $C^{n}$, Ann. Inst. Fourier 30(3) (1980), 219-236.

[5] G. Fischer, Complex analytic geometry. Lecture Notes in Mathematics, Vol. 538. SpringerVerlag, Berlin-New York, 1976.

[6] A. Fujiki, On the blowing down of analytic spaces, Publ. Res. Inst. Math. Sci. 10 (1974/75), $473-507$.

[7] H. Grauert, Über Modifikationen und exzeptionelle analytische Mengen, Math. Ann. 146 (1962), $331-368$.

[8] A. Grothendieck, Techniques de construction en géométrie analytique V. Fibrés vectoriels, fibrés projectifs, fibrés en drapeaux. Séminaire Henri Cartan; 13e année: 1960/61. Exposé no. 12, 15 pp.

[9] R. Hartshorne, Algebraic geometry. Graduate Texts in Mathematics, No. (52) Springer-Verlag, New York-Heidelberg, 1977.

[10] R. Hartshorne, Ample vector bundles, Publ. Math. Inst. Hautes Études Sci. No. 29 (1966), 63-94.

[11] K. Knorr and M. Schneider, Relativexzeptionelle analytische Mengen, Math. Ann. 193 (1971), $238-254$.

[12] R. Narasimhan, The Levi problem for complex spaces. II, Math. Ann. 146 (1962), 195-216.

[13] M. Schneider, Familien negativer Vektorraumbündel und 1-konvexe Abbildungen, Abh. Math. Sem. Univ. Hamburg 47 (1978), 150-170. 
[14] P. Siegfried, Un théorème de finitude pour les morphismes q-convexes, Comment. Math. Helv. 49 (1974), 417-459.

[15] K.-W. Wiegmann, Über Quotienten holomorph-konvexer komplexer Räume, Math. Z. 97 (1967), 251-258.

Simion Stoilow Institute of Mathematics of the Romanian Academy, Research Unit 3 , P.O. Box 1-764, Bucharest 014700, Romania

E-mail address: Mihnea.Coltoiu@imar.ro, Cezar.Joita@imar.ro

Mathematik, Universität Wuppertal, Gausstr. 20, D-42095 Wuppertal, Germany

E-mail address: klas@math.uni-wuppertal.de 